\title{
AUTOMOTIVE HEALTH TRACKING USING IOT AND ARDUINO
}

\author{
Prakhar Prakash
}

\begin{abstract}
This paper is concerned with the development of an embedded system for detecting the vehicle condition by monitoring the internal parameters used in evaluating the vehicle's current health condition. An in-vehicle embedded system is being developed in this project to generate a vehicle health report (VHR) whenever the user requires it. It predicts future errors, allowing the driver to travel without interruption and avoid accidents. As a result, it warns the driver of potential errors and assists him in driving safely. The data needed to generate the health report is made up of parameter values (outputs of in-built sensors) from various systems inside the vehicle. Our framework is based on the Arduino and IoT stages, which are used to separate various parameters, for example, motor warming and fuel pipe blockage, for safe and cautious driving. The data is sent to IoT, where it can be checked by both the vehicle manufacturer via distributed computing and the vehicle owner via the Android application. The equipment unit consists of an Arduino, a WI-FI module, a portable Androidbased device, and a unique parameter checking sensors module. The ESP8266 WiFi Module is a self-contained SOC with integrated TCP/IP protocol stack that can give any microcontroller access to your WiFi network, which is used in current generation automotive.
\end{abstract}

\section{INTRODUCTION}

Recent interest in smart sensor networks and developments in technologies such as MEMS, microelectronics, nanotechnology, communication networks, and distributed computing have fueled interest in the development of vehicle condition monitoring (VHM) systems. In the long term, these systems will form the basis for the development of self-healing and perhaps even timeless structures. In the short term, IVHM could reduce or eliminate several current design constraints (e.g., redundancy and auditability), which should allow for more efficient designs and reduce maintenance and inspection requirements.

In fields such as mining and petroleum industry, maintenance of vehicles used in areas such which are difficult to access (such as underground mines), monitoring of vehicle health using an android application can be useful for proper maintenance and increasing efficiency of the transportation process. Vehicles needs repair after a certain interval of time and if are not repaired at fixed intervals it can lead to loss of life of the person driving the vehicle or travelling on it. The objective of the project is to notify the owner about excessive vibration of the vehicle signaling that the vehicle needs maintenance.

\section{CHALLENGES}

The issues that must be considered in designing an VHM system are the environment in which the vehicle is to operate, the nature of its mission(s) and the threats it will face. Other issues are as follows

- Low communication range of Bluetooth module used

- Network availability for GSM based communication

- As the project deals with vehicles in motion, incorporation of the circuit and sensor in a small volume is a major challenge.

\section{CIRCUIT DIAGRAM}

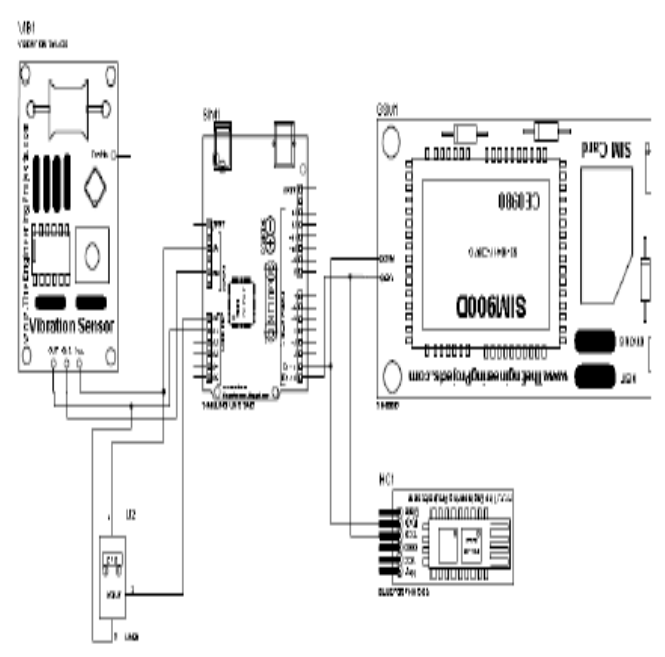




\section{International Journal of Engineering Applied Sciences and Technology, 2021 \\ Vol. 6, Issue 5, ISSN No. 2455-2143, Pages 189-192 \\ Published Online September 2021 in IJEAST (http://www.ijeast.com)}

\section{BLOCK DIAGRAM}

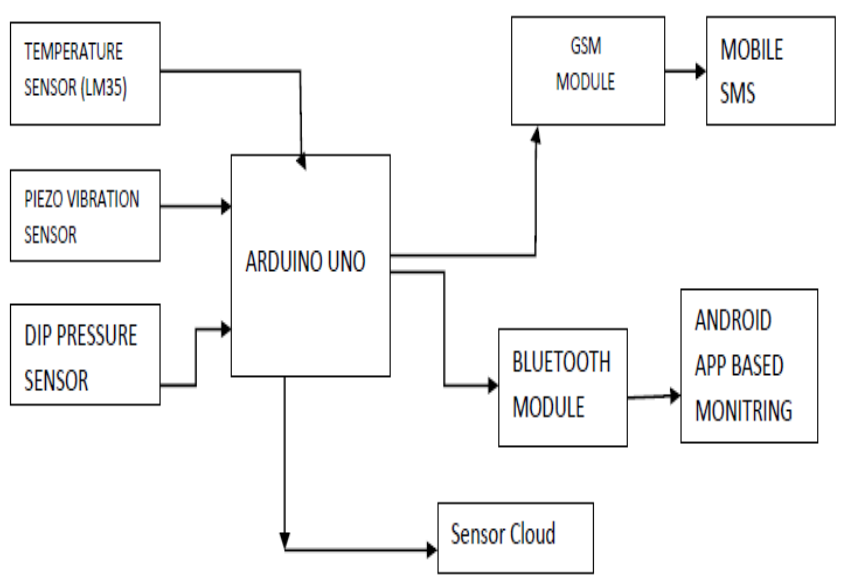

The hardware design consists of

- $\quad$ Arduino UNO

- $\quad$ Temperature sensor (LM35)

- $\quad$ Piezoelectric vibration sensor (LDT0-028K)

- $\quad$ DIP air pressure sensor

\section{Arduino UNO}

The Arduino Uno is a microcontroller board based on the ATmega328. The Uno differs from all preceding boards in that it does not use the FTDI USB to-serial driver chip. Instead, it features the Atmega16U2 programmed as a USBto-serial converter. With the help of this we can directly communicate with the PC or computer. In the system developed we have used Arduino board since it has inbuilt $\mathrm{ADC}$ so there is no need to interface external $\mathrm{ADC}$ to connect with sensor, since most of the sensor gives their output in analog form. Since.

Feature of Arduino Uno board

- $\quad$ Microcontroller ATmega168 or 328

- $\quad$ Operating Voltage 5V

- Input Voltage (recommended) 7-12V

- $\quad$ Input Voltage (limits) 6-20V

- $\quad$ Digital I/O Pins 14

- $\quad$ Analog Input Pins 6

- $\quad$ DC Current per I/O Pin $40 \mathrm{~mA}$
- $\quad$ DC Current for 3.3V Pin $50 \mathrm{~mA}$

- $\quad$ Flash Memory 16 KB (ATmega168) or 32 KB

- $\quad$ (ATmega328) of which $2 \mathrm{~KB}$ used by bootloader

\section{TEMPERATURE SENSOR}

To measure engine temperature, we have used LM35 sensor. It is an industrial temperature sensor which generates high output voltage without need to amplify the output voltage. The output voltage is directly proportional to the Celsius temperature. The scale factor is $0.1 \mathrm{v} / \mathrm{o}$ c. The LM35 does not require any external calibration or trimming to provide typical accuracies of $\pm 1 / 4^{\circ} \mathrm{C}$ at room temperature and $\pm 3 / 4^{\circ} \mathrm{Cover}$ a full -55 to $+150^{\circ} \mathrm{C}$ temperature range. The range of this sensor is $-550 \mathrm{C}$ to $1500 \mathrm{C}$. It is low cost and easily available sensor. It has also low self-heating. LM35 has three terminal VCC, GND, O/P

\section{PIEZOELECTRIC VIBRATION SENSOR(LDT0- 028K)}

Piezo vibration sensor has been used to diagnose vibration of the vehicle body. The LDT0-028K is a flexible component comprising a $28 \mu \mathrm{m}$ thick piezoelectric PVDF polymer film with screen-printed Ag-ink electrodes, laminated to a 0.125 $\mathrm{mm}$ polyester substrate, and fitted with two crimped contacts. As the piezo film is displaced from the mechanical neutral axis, bending creates very high strain within the piezopolymer and therefore high voltages are generated. If the assembly is supported by its contacts and left to vibrate "in free space" (with the inertia of the clamped/free beam creating bending stress), the device behaves as an accelerometer or vibration sensor.

\section{FEATURES}

- Solder Tab Connection

- Both No Mass \& With Mass Version

- Withstands High Impact

- Operating Temperature: $0^{\circ} \mathrm{C}$ to $85^{\circ} \mathrm{C}$

- Storage Temperature: $-40^{\circ} \mathrm{C}$ to $85^{\circ} \mathrm{C}$

- Higher Temperature Version up to $125^{\circ} \mathrm{C}$ available on a Custom Basis

DIP AIR PRESSURE SENSOR 0-40KPA DIP-6 FOR ARDUINO

A pressure sensor has been employed for continuous monitoring of tyre air pressure. This DIP air pressure sensor is suitable for biomedical, meteorology and other fields. The core part is pressure sensor chip which is make by the MEMS pressure technology. The sensor can be installed at the 
pressure valve of the tyre and provides precise pressure readings.

\section{WIRELESS NETWORK SYSTEM DESIGN}

the wireless communication network consists of

- Bluetooth based real time data acquisition

- $\quad$ GSM based SMS alert system for drastic vehicle conditions

Bluetooth is a short-range wireless technology standard used for (using short-wavelength UHF radio waves in the ISM band from 2.4 to $2.485 \mathrm{GHz}$ [3]) fixed and mobile devices and building personal area networks (PANs). The purpose is to provide a standard low complexity, cost, power consumption, and low data rate wireless connectivity over a considerable range.

GSM (Global System for Mobile communications) is a standard developed to describe the protocols for secondgeneration digital cellular networks used by mobile devices. The GSM wireless system utilizes the cellular network to communicate with the distant user using a SIM installed in in built sim port of the GSM module.

\section{SOFTWARE DESIGN}

The android monitoring program of the monitoring system is developed on online app development platform MIT app inventor and is debugged and run on a smartphone with the Android operating system. The design and implementation of the Android monitoring application include two aspects: design of the android APP user interface and android APP functional modules. Two application user interface needs to be designed in this android application, one is used for the control and operation of the application, the other one is used for displaying analog diagnostic information of vehicle. Android smartphone receives the vehicle health information sent by the sensors via its local Bluetooth, then display in real-time.

\section{THINGSPEAK}

ThingSpeak is a free web service that lets you collect and store sensor data in the cloud and develop Internet of Things applications. The ThingSpeak web service provides apps that let you analyze and visualize your data in MATLAB®, and then act on the data. Sensor data can be sent to ThingSpeak from Arduino ${ }^{\circledR}$, Raspberry $\mathrm{Pi}^{\mathrm{TM}}$, BeagleBone Black, and other hardware.

\section{WORKING}

The system utilizes three sensors to monitor the parameters specified above. The temperature sensor is placed near the

engine, pressure sensor is fixed on the pressure valve of the tyre and the vibration sensor is fixed on the card body. These sensors send data continuously to the Arduino using analog pins. The user connects to the Arduino using Bluetooth through an android application. the user continuously receives real time data of engine temperature, vibration of body and air pressure of tire. The application also provides information about car condition such as "condition is OK" or "engine overheating" etc. The application provides a simple and user-friendly interface. To provide a full proof safe monitoring system, the system is also equipped with GSM based SMS sending capabilities in case of extreme conditions when the vehicle demands urgent maintenance.

\section{RESULT}
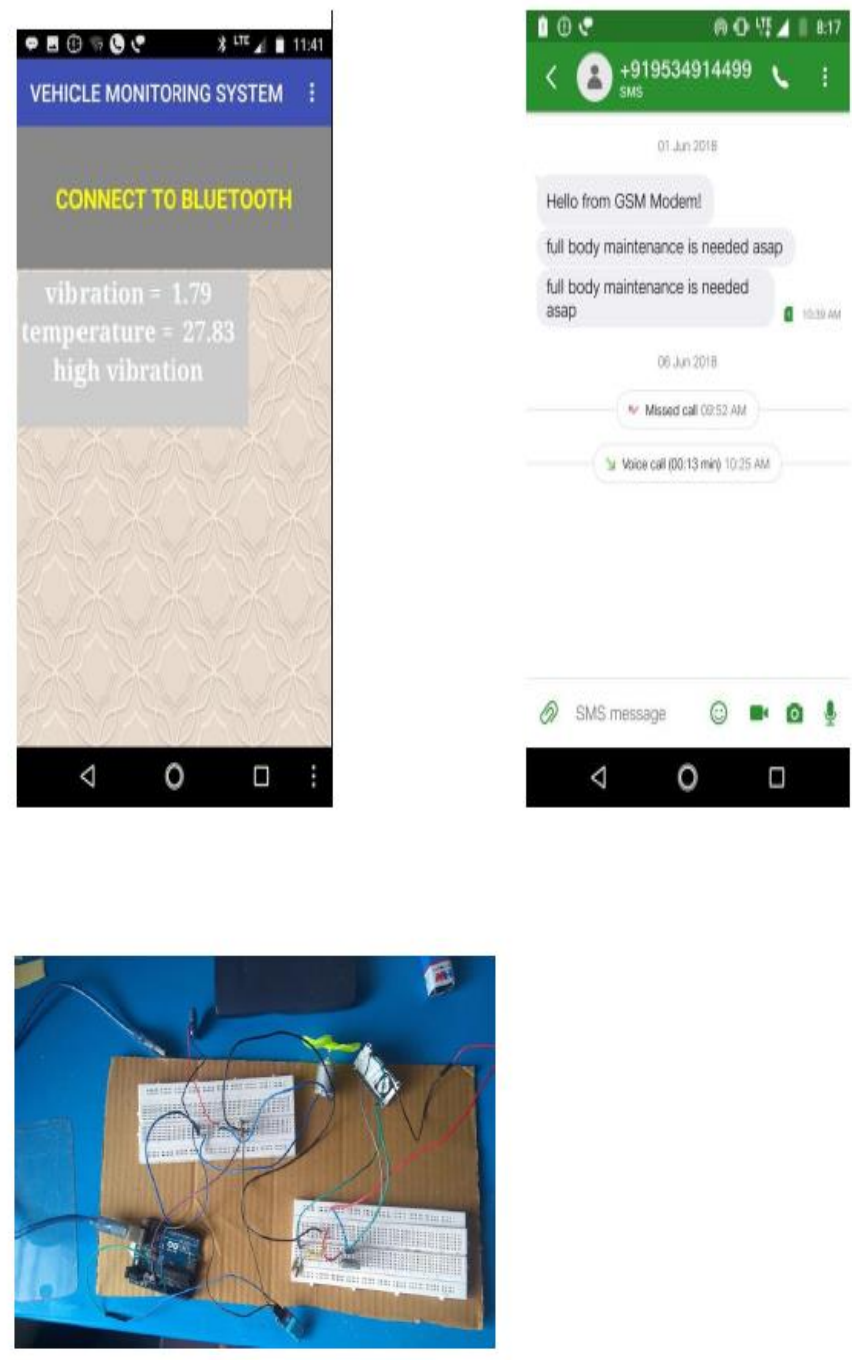

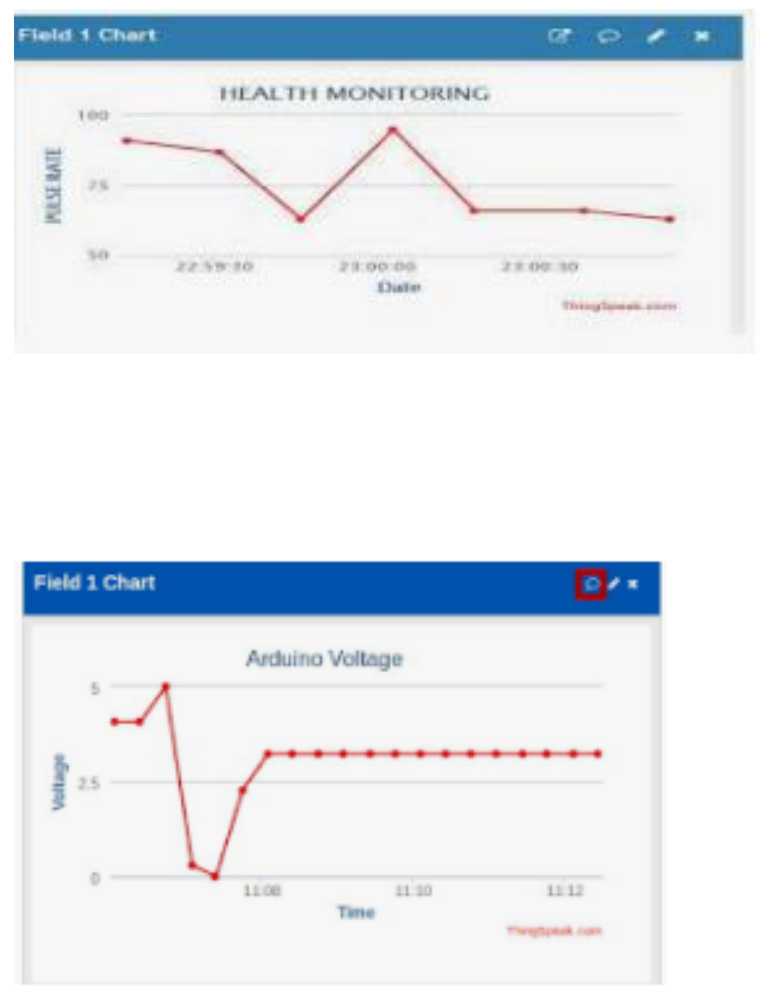

VIII. APPLICATIONS or SCOPE

The Vehicle monitoring system finds widespread use in automobile industry as an instrument to enhance vehicle safety and ensure long life of the vehicle.

- $\quad$ Fuel economy is the major concern today and with the rapid increase in demand for fuel efficient vehicles, the automobile industry will revolve around this. The system provides proper maintenance which reduces fuel consumption to a large extent and thus has a potential to be converted to an in - built device in future cars and vehicles.

- $\quad$ The system also finds use in areas which are difficult to access such as mining sites. These areas employee large vehicles which demand constant maintenance as any breakdown of vehicle may lead to loss of time and money on a large scale.

\section{CONCLUSION}

We have effectively executed a coordinated framework comprising of minimal effort equipment unit and easy to use Android - based portable application to continuously monitor vehicle health parameters in real time and show vehicle condition accordingly. We have also been able to develop a secure system which enables internet availability independent monitoring over the android application and produces a GSM based SMS alert directly to a remote mobile phone in case of conditions where the vehicle demands urgent maintenance

\section{REFERENCE}

[1] Vehicle Health Monitoring Systems. M.Jyothi kiran, S.Ravi teja. International Journal of Engineering Research and Applications Vol. 2, Issue 5, September- October 2012, pp.1162-1167.

[2] Design of Vehicle positioning System Based on ARM, Zhang Wen Jiang Meng, Department of Physics \& Electronic Information Engineering,Neijiang Teachers college, China.

[3] Real Time Vehicle Health Monitoring and Driver Information Display System based on CAN and Android.Esha Naryal,Prabha Kasilwal. International Journal of Advance Foundation and Research in computer Vol. 1, Issue 11, November 2014, ISSN 2348-4853.

[4] Android Based Vehicle Monitoring and Tracking System using ARM7 and CAN technology.T.Narendra kumar,S.Sivaji. International Journal of Engineering and Advance technology,IJSEAT, Vol. 3, Issue 4, April 2015,ISSN 2321-6905. 